\title{
A CNN Based Structured Light Communication Scheme for Internet of Underwater Things Applications
}

\author{
Abderrahmen Trichili*, Chaouki Ben Issaid*, Boon S. Ooi, and Mohamed-Slim Alouini
}

\begin{abstract}
Underwater optical wireless communication is an emerging field that can provide reliable connectivity for future generation internet of underwater things devices. In this paper, we propose a communication system based on single and superposition of Laguerre Gaussian modes to transfer information and rely on a convolutional neural network for the mode identification in an underwater environment. A $100 \%$ recovery fidelity is reported at clear and turbid water. Beyond $90 \%$ of identification, accuracy is achieved under different laboratory-emulated underwater turbulence conditions. The practical implementation of the proposed spatial-mode based communication scheme is further discussed.
\end{abstract}

Index Terms-Underwater wireless optical communication, spatially structured light, index modulation, convolutional neural network, internet of underwater things

\section{INTRODUCTION}

$\mathbf{N}$ EARLY $71 \%$ of the planet's surface is water-covered, and a massive part of the water-occupied surface consists of oceans and seas. So far, only $5 \%$ of the underwater world has been explored. The remaining mysterious part has a high impact on the climate, oxygen production, and carbon absorption. The underwater world equally offers a wide variety of opportunities for renewable energy generation, bio-ocean farming, and food production. Underwater scientific activities such as climate change tracking, pollution control, seismic surveys, and marine life monitoring are also continuously growing, and in this regard, efficient underwater communication systems are needed. Underwater communication is equally of interest for several human activities, including surveillance and protection, off-shore oil and mineral exploration, and aquaculture. Delivering information underwater can be performed through three different waves, which are the acoustic, radio frequency, and optical waves. For more than half a century, acoustic communication has been a technology of choice to communicate underwater. Acoustic waves are utilized to carry information over multi-kilometer distances through sea and ocean waters, typically for frequencies between $10 \mathrm{~Hz}$ and $1 \mathrm{MHz}$ [1]. However, acoustic communication technology suffers from low bandwidth [2], and because of the low propagation speed of acoustic waves through the water, this technology suffers from huge latencies. Furthermore, acoustic

Authors are with the Computer, Electrical and Mathematical Sciences \& Engineering division of King Abdullah University of Science and Technology (KAUST), Thuwal, Makkah Province, Kingdom of Saudi Arabia. email:\{abderrahmen.trichili, chaouki.benIssaid, boon.ooi, slim.alouini\}@kaust.edu.sa. *These authors contributed equally to the paper. communication can increase noise pollution for marine life, which can significantly affect different marine species [3].

$\mathrm{RF}$ waves offer an extremely higher velocity in the water than the acoustic ones. However, RF signals are highly attenuated in seawater and cannot reach long distances unless at the super-low frequency band, i.e., from 30 to $300 \mathrm{~Hz}$. Communicating over this frequency band requires sophisticated large antennas that entail a large amount of transmit power, which limits the practicability of such an approach [4].

Underwater wireless optical communication (UWOC) offers a large unlicensed bandwidth that has led to the successful transfer of data signals with rates beyond one Gbps over short and moderate propagation distances [5], [6]. Due to the high velocity of lightwaves in the water, UWOC can securely carry information without latency. UWOC is also seen as a new way to deliver power to the future internet of underwater things (IoUT) devices by transferring power along with the information to remote devices [7]. Considerable progress has been made so far in the field of UWOC, and different techniques have been introduced to increase the reliability and transmission capacity of underwater links [8].

One way to increase UWOC data rates is to encode information signals over orthogonal spatial modes of light, as demonstrated in [9]-[11]. The concept consists of using the spatial structure of light as an extra degree of freedom for communication systems. A particular mode of choice for spatial multiplexing is the orbital angular momentum (OAM) [12]. OAMs form a subset of the Laguerre Gaussian (LG) mode family, which is detailed in section III-A. Several approaches have been proposed in the literature to generate and detect OAM and LG beams in general. The generation can be mainly performed using computer-generated holograms (CGHs) programmed on spatial light modulators (SLMs) [13] or through spiral phase plates (SPPs) [14]. The detection of LG modes can be performed via CGHs [15], [16] or using mode sorters [17], [18]. These LG mode detection techniques are bulky and require maintaining strict system alignment to minimize intermodal crosstalk and recover the initially encoded information on the carrier modes. However, UWOC links can be subject to different kinds of turbulence related to the underwater conditions, which can severely affect the pointing between the transmitter and the receiver.

One potential solution to cope with the effects of turbulence for underwater spatial mode links is to rely on adaptive optics (AO) to correct distortions at the beam level, as suggested in [19], and demonstrated in free space optics (FSO) [20]. 
Also, similarly to FSO, using digital signal processing (DSP) techniques, it is possible to undo the effect of turbulence [21]. Although AO and DSP methods have shown to be of high performance, implementing such techniques can be costly or may lead to an increase in system complexity [12]. However, using the transverse patterns of spatially structured light beams, such as the LG, as code-words can be a way to deliver information in turbulent underwater channels without the need to compensate for propagation effects through AO or DSP techniques. The concept can be defined as a mode index modulation method that involves the shapes of single modes as well as combinations of modes to transfer information. A particular approach to distinguish between the transverse intensity patterns is to use a machine learning algorithm, and a camera, instead of state-of-the-art alignment-sensitive mode detection approaches. In this context, we propose the use of LG mode patterns to communicate underwater and use a convolutional neural network $(\mathrm{CNN})$ to identify the modes propagating through a turbulent channel. The construction of the proposed $\mathrm{CNN}$ is based on the use of the transfer learning technique [22], [23] of the pre-trained neural network ResNet34 [24]. The use of transfer learning allows us to accelerate the training process since the neural network is trained using a certain prior knowledge rather than starting the learning from scratch. The basic idea behind this technique is to use the knowledge acquired by a neural network when solving a problem in order to solve another more or less similar, which corresponds to a transfer of knowledge, hence the name.

The remainder of this paper is organized as follows. Section II is dedicated to the related published work in the literature. In Section III, preliminaries needed to understand the presented contribution are provided. Section IV is devoted to the experimental demonstrations where the different steps to transmit information using spatially structured beams through the water under different channel conditions are described. Results of the identification of modes are presented and discussed in Section V. A discussion on the practical implementation and the potential applications of the proposed communication technique is included in Section VI. Finally, the paper concludes by proposing new research directions.

\section{RELATED WORK}

The concept of structured light beam detection using machine learning algorithms has been a topic of interest in free space optics. Krenn et al. implemented an artificial neural network to identify the intensity patterns of a Gaussian beam profile and 15 petal-like beams (from $\ell= \pm 1$ to $\ell= \pm 15$ ) after propagating for 3 kilometers through turbulent atmosphere across the city of Vienna [25]. A similar approach was used to recognize the transverse profiles of 4 OAM beams and 4 OAM superpositions, with high identification accuracy that exceeded $80 \%$, over a propagation distance of $143 \mathrm{~km}$ between two Canary islands [26]. A study by Knutson et al. demonstrated the potential of machine learning algorithms for the identification of high-charge OAMs [27]. Authors of [28] demonstrated that a CNN-based algorithm allows differentiating with a precision rate of $99 \%$ between the combination of
OAM beams when propagating through laboratory-emulated highly-turbulent free space links. Zhao et al. suggested the use of a CNN-based approach for high-accuracy identification of up to 10 superposition of OAM beams subject of tilting and lateral displacements caused by atmospheric turbulence effects simulated using the modified Kolmogorov turbulence model [29]. In a theoretical study, Tian et al. proposed a turbo-coded 16-OAM shift-keying (OAM-SK) communication scheme for FSO systems subject to turbulence, which is based on a CNN for mode identification [30]. In a recently published survey [12], we reviewed the main contributions on the potential of machine learning algorithms for the detection of structured light modes, and we highlighted the potential of these techniques to relieve the strict alignment requirement of state-of-the-art detection techniques.

Here, we propose using the intensity patterns of a set of single and superposition of LG beams as underwater information carriers. A CNN-based method is implemented to recover the initially transmitted modes instead of using previously proposed alignment-sensitive projection techniques and bulky mode sorters. Different underwater channel conditions are considered, and the potential of our encoding and decoding technique is assessed in each case.

\section{PRELIMINARIES}

In this section, we give an introduction to spatially structured light modes from the LG mode family. We equally propose two encoding techniques that can be performed with an SLM and used to transmit information using the LG mode profiles as code-words. Then, we provide an overview of underwater propagation and the different approaches to model the propagation effects in particular for structured light. We finally provide a detailed description of the convolutional neural network that will be used for mode identification.

\section{A. LG Modes}

LG modes form a set of solutions to the paraxial Helmholtz wave equation. Each mode is characterized by two degrees of freedom $\ell$ and $p$, known respectively, as the azimuthal and radial indices. $\ell$ is responsible for the OAM and defining the twist of the helical phasefront of the wave, and $p$ indicates the radial components. In a cylindrical coordinate system, with a position vector $(\rho, \phi, z)$, the electric field of an LG mode is expressed as follows [31]:

$$
\begin{aligned}
E_{(p, \ell)}^{L G}(\rho, \phi, z)= & \frac{1}{\omega(z)} \sqrt{\frac{2 p !}{\pi(|\ell|+p) !}} \exp (i(2 p+|\ell|+1) \Phi(z)) \\
& \times\left(\frac{\sqrt{2} \rho}{\omega(z)}\right)^{|\ell|} L_{p}^{|\ell|}\left(\frac{2 \rho^{2}}{\omega(z)^{2}}\right) \\
& \times \exp \left(-\frac{i k \rho^{2}}{2 R(z)}-\frac{-\rho^{2}}{\omega(z)^{2}}+i \ell \phi\right),
\end{aligned}
$$

where $\omega(z)=\omega_{0} \sqrt{1+\left(z / z_{R}\right)^{2}}$ is the beam spot size as a function of $z$, the beam waist $\omega_{0}$ and the Rayleigh size $z_{R}=\pi \omega_{0}^{2} / \lambda$ with $\lambda$ being the optical wavelength. $\Phi(z)=$ 
$\arctan \left(z / z_{R}\right)$ denotes the Gouy phase, $R(z)=z\left[1+\left(z / z_{R}\right)^{2}\right]$ is the beam curvature and $L_{p}^{|\ell|}($.$) are the generalized Laguerre$ polynomials. LG modes form a complete mode basis that spans an infinite-dimensional Hilbert space. Two modes with distinct $\ell$ or $p$ indices and having the same beam waist $\omega_{0}$ are orthogonal to one another. The orthogonality is expressed through the inner product operator \langle|$>$ as follows:

$$
\begin{aligned}
H \triangleq & <E_{\left(p_{1}, \ell_{1}\right)}^{L G}(\rho, \phi, z) \mid E_{\left(p_{2}, \ell_{2}\right)}^{L G}(\rho, \phi, z)> \\
& =\iint E_{\left(p_{1}, \ell_{1}\right)}^{L G}(\rho, \phi, z) E_{\left(p_{2}, \ell_{2}\right)}^{L G *}(\rho, \phi, z) \rho d \rho d \phi \\
& =\delta_{\ell_{1}, \ell_{2}} \delta_{p_{1} p_{2}} \iint\left|E_{\left(p_{1}, \ell_{1}\right)}^{L G}(\rho, \phi, z)\right|^{2} \rho d \rho d \phi,
\end{aligned}
$$

where $*$ denotes the complex conjugate.

A linear combination $U=\sum_{n} c_{n} E_{(p, \ell)}^{L G}(\rho, \phi, z)$ of LG modes is also orthogonal to single modes. The mode coefficients, $c_{n}$, can be determined by an inner product of the optical field $U$ and the corresponding mode field distribution in the following manner:

$$
c_{n}=\frac{1}{I}<U \mid E_{(p, \ell)}^{L G}>
$$

where $I$ is the intensity of the $E_{(p, \ell)}^{L G}$.

The phase and intensity profiles of a set of LG modes are
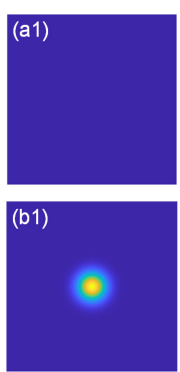
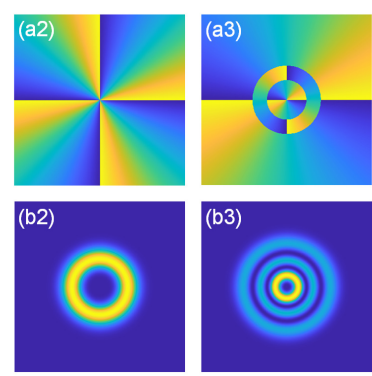

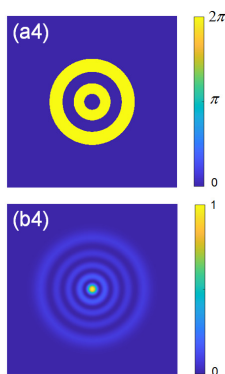

Fig. 1: (a1-a4) Phase profiles of $\mathrm{LG}_{0,0}, \mathrm{LG}_{0,4}, \mathrm{LG}_{2,2}, \mathrm{LG}_{4,0}$ modes, respectively, and (b1-b4) are the corresponding intensity profiles.

shown in Fig. 1.

\section{B. Concept of Mode Index Modulation}

The principle idea is to encode information using the spatial structure of the LG modes and detect the mode profiles using a machine learning algorithm. Two encoding techniques are proposed:

1) Single Mode Encoding: At the transmitter, the input data is a $m \times n$ image with $N$ levels of gray, each gray level is assigned to a single or a superposition of LG modes as illustrated in Fig. 2.

At the receiver, the modes are identified one by one through a convolutional neural network algorithm, which will be detailed in the next section.

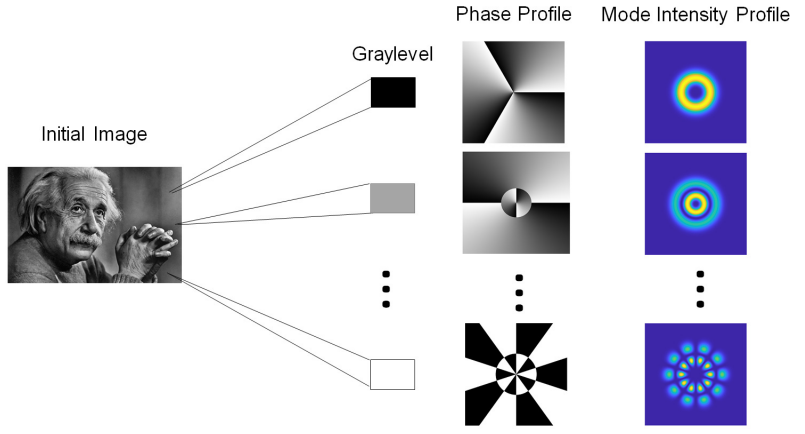

Fig. 2: Concept of single mode information encoding.
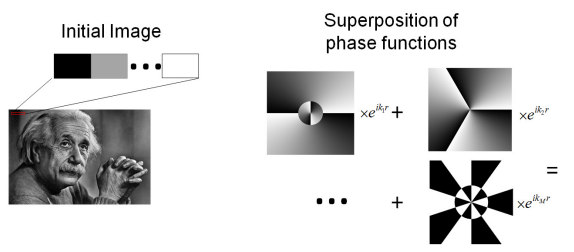

Generated spatially separated modes

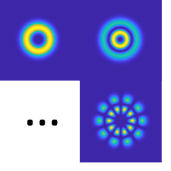

Fig. 3: Concept of the multimode encoding technique.

2) Multimode Encoding: This approach consists of loading a superposition of single transmission function $g_{m}(r)$ on the SLM screen, each is weighed with a particular spatial carrier frequency $k_{m}$ :

$$
T_{S L M}(r)=\sum_{M} g_{m}(r) \exp \left(i k_{m} r\right),
$$

where $M$ denotes the number of multiplexed mode structures. The spatial frequencies $k_{m}$ act as spatial coordinates in the Fourier plane. With a two dimensional image as an input, $M$ pixels can be simultaneously transmitted using spatially separated modes as depicted in Fig. III-B2. At the receiver, each mode is identified separately, and the initially transmitted information is recovered.

\section{Underwater Propagation}

According to Beer's law, the intensity, $I(z)$, of a light beam propagating through the water along the $z$ direction decays exponentially, as follows:

$$
I(z, \lambda)=I_{0} \exp (-c(\lambda) z),
$$

where $c(\lambda)$ is a wavelength $\lambda$-dependent attenuation coefficient. The attenuation coefficient is the contribution of an attenuation coefficient $\alpha(\lambda)$ and a scattering coefficient $b(\lambda)$. In addition to attenuation and scattering, a light beam can also be affected by air-bubbles that can be potentially produced by the breaking of surface waves that infuse the air from the atmosphere into the top layer of the water [32]. When propagating through bubbly water, laser beams get partially or completely obstructed depending on the sizes of the bubbles and the beam radius [33]. This could lead to a deep fading in the received signal as observed in [33]. Temperature and salinity in-homogeneity induce random changes in the refractive index of the water and significantly affect communication quality. A unified statistical model of fading 
induced by air-bubbles and a temperature gradient in fresh and salty water is proposed in [34]. The model was mainly developed for Gaussian-profile beams. Different experimental studies on spatial modes considered underwater turbulence and reported significant impacts on the mode purity over different turbulence regimes [35]. The effect of turbulence has also been theoretically investigated in recent reports [36], [37]. Still, the theoretical background for close-to-reality analytical estimation of the impact of different kinds of turbulence on spatially structured beams requires further efforts [12]. Nonetheless, it is possible to numerically estimate the impact of underwater conditions on spatial modes utilizing random turbulence phase screens. The idea is to assume that the effect of turbulence on the propagating beam is an accumulation of many perturbing planes; a phase screen represents each plane. The turbulence phase screens can be generated using models for the refractive index power spectrum density (PSD) fluctuations. One particular model for oceanic turbulence was proposed in [38], and the PSD of the refractive index, $\Phi_{n}(\kappa)$ is given as follows:

$$
\begin{aligned}
\Phi_{n}(\kappa)= & 0.388 \times 10^{-8} \varepsilon^{-1 / 3} \chi_{T} \kappa^{-11 / 3}\left[1+2.35(\eta \kappa)^{2 / 3}\right] \\
& \times\left(\omega^{2} e^{-A_{T} \delta}+\omega^{-2} e^{\left.-A_{S} \delta\right)}-2 \omega^{-1} e^{-A_{T S} \delta}\right),
\end{aligned}
$$

where $\kappa=\sqrt{\kappa_{x}^{2}+\kappa_{y}^{2}}$ is the wavenumber with $\left(\kappa_{x}^{2}, \kappa_{y}^{2}\right)$ being spatial frequencies in the $\kappa$ space. $\varepsilon$ is the dissipation rate of kinetic energy per unit mass of the fluid, which ranges from $10^{-1}$ and $10^{-10} \mathrm{~m}^{2} / \mathrm{s}^{3} . \chi_{T}$ is the dissipation rate of meansquared temperature. $\eta \mathrm{mm}$ is the inner scale of turbulence (typically equals to $1 \mathrm{~mm}$ ). $A_{T}=1.896 \times 10^{-2}, A_{S}=1.9 \times 10^{-4}$, $A_{T S}=9.41 \times 10^{-3}$, and $\delta=8.284(k \eta)^{4 / 3}+12.978(k \eta)^{2}$. $\omega$ is a unitless quantity that measures the relative strength of temperature and salinity induced fluctuations and ranges from -5 to 0 ( -5 when the temperature is dominating and 0 when salinity is dominating). It is possible to simulate the propagation through turbulent water using the split-step Fourier method following [39].

\section{Convolutional Neural Network}

A neural network is a mathematical model whose design is inspired by the functioning of biological neurons. It is a system composed of neurons [40], generally divided into several interconnected layers. A neuron performs a scalar product between the input values and its parameters (weights) and then applies a function to the result. Such a system is used to solve various statistical problems. For the problem of classification, the network computes from the inputs a vector containing the probabilities of belonging to each class. The class assigned to the input object corresponds to the class with the highest probability. For image classification tasks, convolutional neural networks are considered today to be the best performing models for this type of application [41] [42]. CNNs have a methodology similar to traditional methods of supervised learning. In fact, they receive inputs in the form of images, detect the features of each of them, and then train a classifier on them. However, the features are learned automatically. CNNs do all the hard work of the extraction and the description of the features. During the training phase, the classification error is minimized in order to optimize the parameters of the classifier and the features. In addition, the specific architecture of the network can extract features of different complexities, from the simplest to the most complex. For instance, a $99.77 \%$ accuracy was obtained using CNN on the handwritten digit MNIST dataset [43], a 97.47\% correct detection rate with the $3 \mathrm{D}$ object NORB dataset [44], and a $97.6 \%$ accuracy on face recognition task [45]. In comparison to other detection algorithms, CNNs not only provide the greatest performance but also exceed human capacity for cases like the classification of objects into finer categories like dog or bird species [46].

Each convolution kernel in one layer of the CNN produces a different feature map so that the output neurons are different for each kernel. The convolutional networks may also include local or global "pooling" layers [47]. The combination of the outputs may, for example, consist of taking the maximum or the average value across the "pooled" area. The "pooling" layers make it possible to reduce the size of the feature map from one layer to another in the network while improving its performance by making it more tolerant for small deformations or translations in the input data. The values of the weights of the layers are learned by backpropagation of the gradient [48] [49]. In fact, for each layer, starting from the end of the network, the parameters, which minimize the loss function, are computed gradually, using gradient descent, for instance. The last layer computes the final probabilities using the softmax function as an activation function since the task at hand is a multi-class classification problem. In fact, the softmax function will return the output in the form of a vector having the same size as the number of classes. Each element of the vector indicates the probability for the input image to belong to a class. For a vector $x=\left[x_{1}, x_{2}, \ldots, x_{N}\right]$, the softmax function outputs the vector $y=f(x)$ where each component of $y$ is defined as

$$
y_{i}=\frac{e^{x_{i}}}{\sum_{j=1}^{N} e^{x_{j}}}
$$

As we have mentioned, the class assigned to the input will be the one having the highest probability. A loss function is associated with the final layer to calculate the classification error. In our case, this loss function happens to be the categorical cross-entropy defined as

$$
\mathcal{L}(y, \hat{y})=-\frac{1}{n} \sum_{i=1}^{n} \sum_{j=1}^{m} y_{i j} \log \left(\hat{y}_{i j}\right),
$$

where $i$ denotes the observations and $j$ indexes the classes, and $y$ is the sample label while $\hat{y}_{i j} \in(0,1)$ such that $\forall i$, we have $\sum_{j=1}^{m} \hat{y}_{i j}=1$ is the prediction for the $i^{t h}$ observation.

Since the task of image classification is a very complex task that requires training the neural network for lengthy hours, transfer learning technique can be seen as a practical solution to this problem. In fact, by using knowledge of models already trained on more or less similar tasks, transfer learning can help reduce the amount of training considerably and achieve excellent performance results. 
(a)

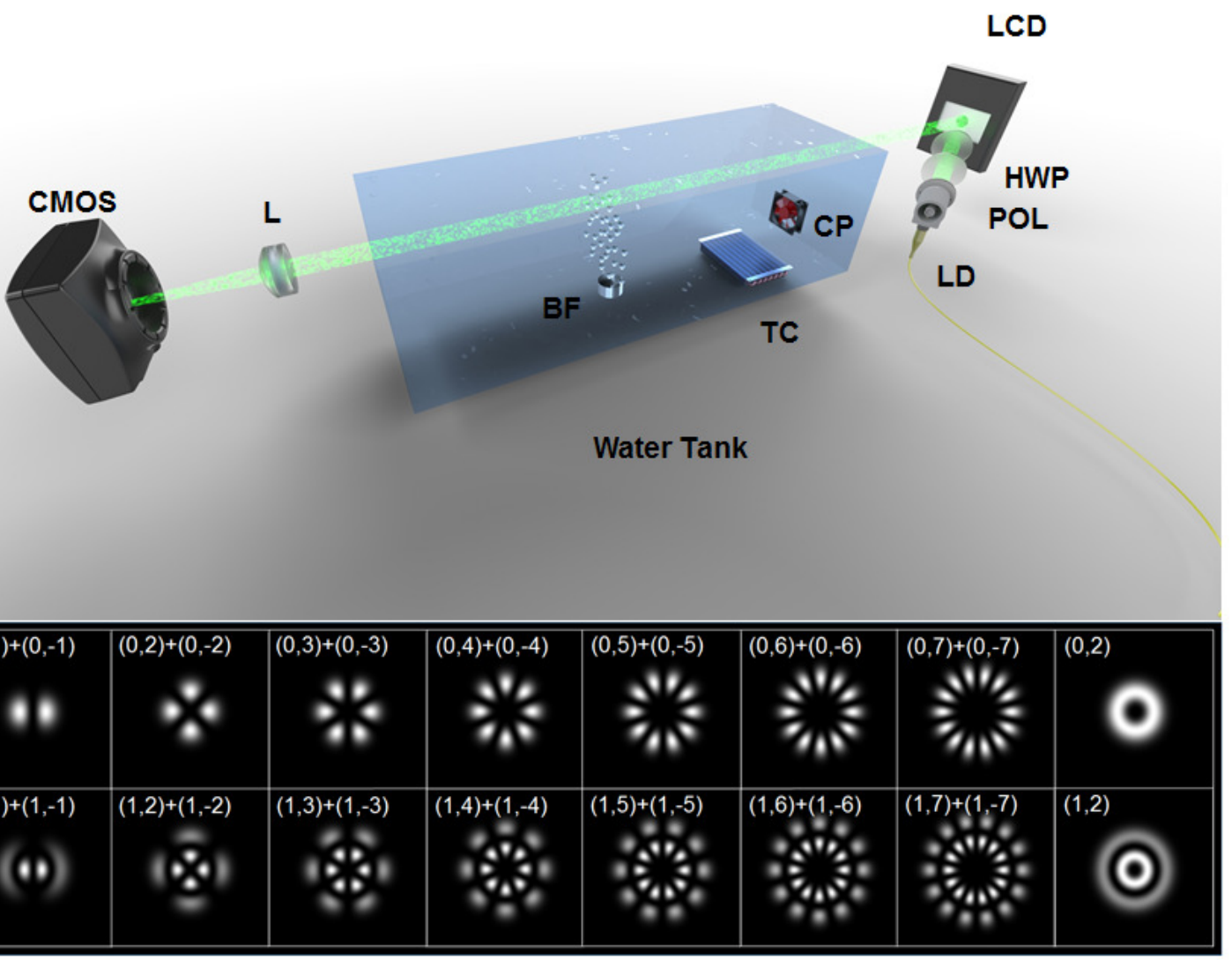

Fig. 4: (a) Experimental setup for LG mode encoding and detection. LD: Laser diode; HWP: half-wave plate; POL: linear polarizer; LCD: liquid crystal display (of an SLM); CP: circulation pump; TC: temperature controllable chiller; BF: Bubble Fountain; L: lens; CMOS: camera. (b) LG mode profiles forming the communication alphabet.

\section{EXPERIMENTAL IMPLEMENTATION}

The experimental setup to encode information using LG modes is depicted in Fig. 4 (a). The transmitter is composed of a green light single-mode fiber pigtailed laser (Thorlabs LP520-SF15) directed to a $1920 \times 1080$ liquid-crystal display (LCD) of a phase-only SLM (PLUTO2 NIR-011, having a pixel pitch of $8 \mu \mathrm{m}$ ). Since the used SLM is polarization sensitive, in order to adjust the polarization of the emitted light beam to be matched with the optimal polarization state of the LCD, a $\lambda / 2$ plate and a linear polarizer are placed after the laser. The produced LG beams are then propagated through a $1.5 \mathrm{~m}$ water (from the tap) tank. At the reception, we utilize a CMOS camera (DCC1645C by Thorlabs) to receive the transmitted mode patterns.

The images are encoded pixel by pixel. Here we only consider the single mode encoding case, and in each time, a particular hologram is loaded onto the LCD of the SLM to generate a particular pattern as previously explained in sub-section III-B and illustrated in Fig. 2.

In our experimental demonstrations, we consider the propagation through clear water, turbid water, water with temperature in-homogeneity, and bubbly water. To emulate turbidity in the water tank, we fixed a circulation pump, on a position marked as $\mathrm{CP}$ in Fig. 4 (a), which creates a sea-like water current that can be compared to the situation of deepwater conditions. In order to create air-bubbles over the propagation path, a bubbling fountain connected to a $N_{2}$-gas tube is placed in the middle of the water tank. A controllable chiller is installed on the tank to create the temperature in-homogeneity. Note that the temperature of the chiller is continuously rising when it is operational, and the circulation pump is also switched 'on' to create the uniformity of the temperature inside the tank.

The patterns forming the communication alphabet are depicted in Fig. 4. The modes are denoted as $\mathbf{M}_{N}\left(\mathrm{M}_{1}\right.$ : $\mathrm{LG}_{0,1}+\mathrm{LG}_{0,-1}, \quad \mathrm{M}_{2}: \quad \mathrm{LG}_{0,2}+\mathrm{LG}_{0,-2}, \quad \mathrm{M}_{3}: \quad \mathrm{LG}_{0,3}+\mathrm{LG}_{0,-3}$, $\mathrm{M}_{4}: \mathrm{LG}_{0,4}+\mathrm{LG}_{0,-4}, \mathrm{M}_{5}: \mathrm{LG}_{0,5}+\mathrm{LG}_{0,-5}, \mathrm{M}_{6}: \mathrm{LG}_{0,6}+\mathrm{LG}_{0,-6}$, $\mathrm{M}_{7}: \mathrm{LG}_{0,7}+\mathrm{LG}_{0,-7}, \mathrm{M}_{8}: \mathrm{LG}_{0,2}, \mathrm{M}_{9}: \mathrm{LG}_{1,1}+\mathrm{LG}_{1,-1}, \mathrm{M}_{10}$ : $\mathrm{LG}_{1,2}+\mathrm{LG}_{1,-2}, \mathrm{M}_{11}: \mathrm{LG}_{1,3}+\mathrm{LG}_{1,-3}, \mathrm{M}_{12}: \mathrm{LG}_{1,4}+\mathrm{LG}_{1,-4}, \mathrm{M}_{13}$ : $\mathrm{LG}_{1,5}+\mathrm{LG}_{1,-5}, \mathrm{M}_{14}: \mathrm{LG}_{1,6}+\mathrm{LG}_{1,-6}, \mathrm{M}_{15}: \mathrm{LG}_{1,7}+\mathrm{LG}_{1,-7}, \mathrm{M}_{16}$ : $\mathrm{LG}_{12}$ ). The measured profiles of an $\mathrm{LG}_{12}$ beam propagating through different kinds of underwater conditions are depicted in Fig. 5. Figs. 5(a1-a4) show the intensity profile of an $\mathrm{LG}_{1,2}$ at clear water, which corresponds to the situation when no flow is applied. As can be seen in Figs. 5(b1-b2), due to the effect of air-bubbles, the beam can be partially or almost completely obstructed. Under a temperature in-homogeneity regime, the beam shape is distorted and varies during the time, as can be seen in Figs. 5(c1-c4). Turbidity in water with homogeneous temperature and with no bubbles, does 


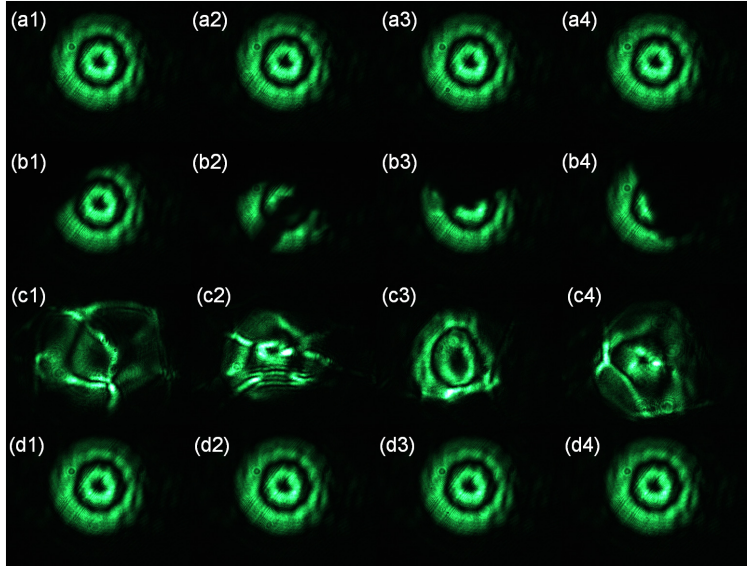

Fig. 5: Measured intensity transverse profiles of an $\mathrm{LG}_{12}$ mode (a1-a4) in clear water, (b1-b4) under an air-bubbles regime, (c1-c4) under temperature inhomogeneity, and (d1-d4) through turbid water.

not seem to change the beam shape over time, as can be seen in Figs. 5(d1-d4).

\section{RESUlts AND Discussion}

Using the experimental setup shown in Fig. 4(a), we generate a dataset containing mode patterns captured under different channel conditions: clear water, turbulent water, bubbly water, and water with temperature inhomogeneity. The dataset contains an equal number of images from each of the 16 modes forming the encoding alphabet (Fig. 4(b)). The total number of images is $400 \times 16=6400$ images split into $70 \%$ for the training and $30 \%$ for the validation. The training set is used to train a model, and the validation set is used to tune the model hyperparameters and to give us an idea about the model performance.

We have performed data augmentation for the training set by applying geometrical deformations to the images, including horizontal and vertical symmetry, rotation, and zooming, etc. This allows our model to distinguish invariant characteristics better and also to increase the number of images with the same concepts for better learning results. All images are resized to have a $224 \times 224$, the size of ImageNet dataset images [50], which were used to train ResNet34. A batch size of 32 was used to update the weights of the neural network, and the images were also normalized using the ImageNet dataset statistics. This model is a CNN trained on the ImageNet dataset, which contains 1.28 million images of 1000 different categories. After a first layer consisting of one convolution and a pooling step, the model has four blocks of layers. Each block is formed by a set of $3 \times 3$ convoluted layers stacked on top of each other with a fixed feature map dimension along with applying batch normalization and a ReLU activation function where its output is defined as the maximum between the input value and zero. The feature map dimensions are 64, 128,256 , and 512 , respectively, bypassing the input every two convolutions. The number of layers in each block is $6,8,12$, and 6, respectively. Each of these layers focuses on a graphic element to be identified, from the most general to the most particular. Weights will be defined for each one, thus making it possible to rank the combinations that will identify the most approximate global form of the object to be found. After these four blocks of layers, we find a global average pooling layer and a fully connected layer containing 1000 nodes. This final layer has as outputs the scores of the 1000 categories on the ImageNet dataset. By applying the softmax activation function, these scores are then transformed into probabilities for each possible output. For the implementation of our network, we have used Fastai [51], with PyTorch [52] under the hood. Our choice is justified by the fact that Fastai allows for easy construction and fast training of deep neural networks.

For the splitting of the dataset into training and validation, we include all the different modes, corresponding to images taken under different conditions, with the same proportion into the training and the validation sets to ensure that we have a balanced dataset and that the various conditions are taken into consideration in both the training and the validation.

In addition to speeding up the training of the network, transfer learning makes it possible to avoid overfitting. In fact, when the dataset is small, it is strongly disadvised to train the neural network from scratch, i.e., that is to say with a random initialization, the number of parameters to learn to be much higher than the number of images, the risk of overfitting is huge in this case. In this work, we have used a total fine-tuning strategy. We have replaced the fully-connected layer of the pre-trained network by a fully connected layer containing 16 nodes, which corresponds to our mode classification problem. All layers are then trained using the training dataset. As the parameters of all the layers, except those of the last one, are initially those of the pre-trained ResNet34, the learning phase will be done more quickly than if the initialization had been random.

To minimize the time needed to determine a good starting learning rate experimentally without the need to re-train the CNN many times, we used the idea of cyclical learning rates [53]. We start by training the neural network for a few iterations while changing the learning rate after each minibatch. We start by a very low learning rate, $\alpha=10^{-7}$, until we reach a high learning rate, $\alpha=10$ and we plot the curve of loss (instead of the accuracy as explained in [53]) at each iteration as a function of the learning rate, in log scale, as shown in Fig. 6. The best optimal learning rate is chosen approximately in the middle of the deepest descent slope. This value corresponds to the point where the gradient is the steepest. In our case, this corresponds to a value of learning rate equal to $\alpha=5.75 \times 10^{-4}$. Using this value, we train the model over four epochs using the one cycle policy [54]. This means that the learning rate and the momentum during the batch normalization process vary as follows:

- In the first step, we increase the learning rate from $\frac{\alpha}{D}$ to $\alpha$ and decrease the momentum from a maximum value to a minimum value. Here, $D$ is known as the division factor, taken to be equal to 25 in our experiment. The maximum (respectively minimum) value of the momentum is 0.95 
(respectively 0.85 ).

- In the second step, we decrease the learning rate from $\alpha$ to $\frac{\alpha}{D}$, and increase the momentum from 0.85 to 0.95 .

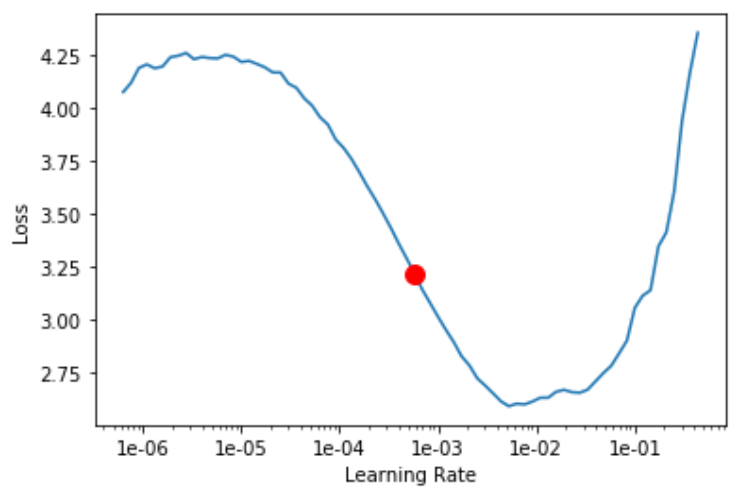

Fig. 6: Cyclical leaning rate: Loss as a function of the learning rate.

In Fig. 7 (respectively Fig. 8), we show how the learning rate (respectively the momentum) varies as a function of the number of iteration. The idea is that we want to the optimization algorithm to take fast steps (increase the learning rate and decrease the momentum) towards the minimum in the first phase, but as we approach the minimum value, we want to take small steps instead (decrease the learning rate and increase the momentum).

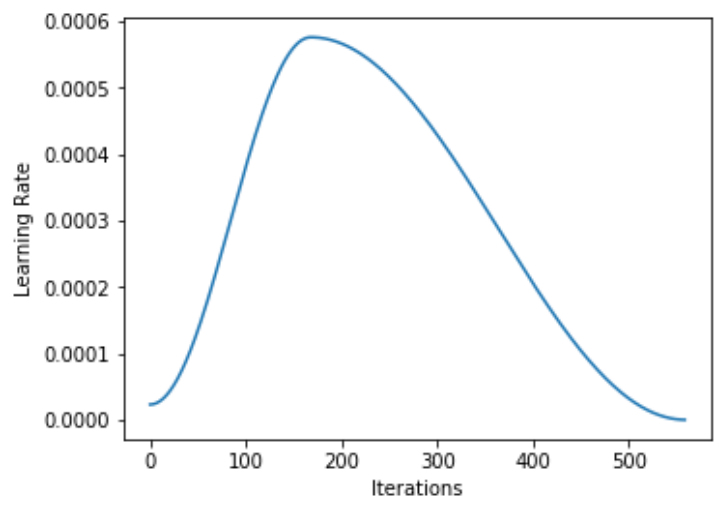

Fig. 7: Leaning rate as a function of the number of iterations (one cycle policy).

The training and validation losses, as well as the validation accuracy at the end of each epoch, are reported in Table I. The loss of training and validation decreases with the number of epochs, and this reflects that in each epoch, the model learns more information. By increasing the number of epochs, we are teaching the model more information; therefore, the loss decreases. Similarly, the validation accuracy increases with the number of epochs, and we reach $100 \%$ accuracy at the fourth epoch.

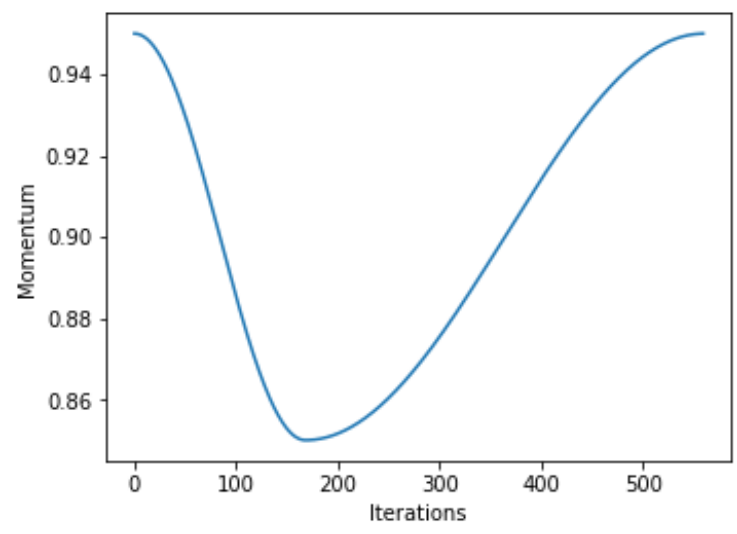

Fig. 8: Moment as a function of the number of iterations (one cycle policy).

TABLE I: Training and validation losses and validation accuracy per epoch.

\begin{tabular}{cccc} 
Epoch & Train Loss & Validation Loss & Validation Accuracy \\
\hline \hline 1 & 0.47 & 0.58 & 0.87 \\
2 & 0.17 & 0.03 & 0.9 \\
3 & 0.05 & $2.3 \times 10^{-4}$ & 0.99 \\
4 & $7 \times 10^{-3}$ & $2.3 \times 10^{-5}$ & 1
\end{tabular}

Using the single-mode encoding technique, we transmitted through different underwater conditions, the $77 \times 75$ grayscale image depicted in Fig. 9(a). Four datasets are now formed, each dataset contains 5775 mode profiles that correspond to the total number of pixels of the 'Einstein image', taken each under the same channel conditions. At clear water, the initially sent image was perfectly reconstructed as seen in Fig. 9(b). A $100 \%$ recovery fidelity is obtained in the case of turbid water propagation, as shown in Fig. 9(c). The received image through a bubbly channel is depicted in Fig. 9(d), and the error rate is equal to $9.73 \%$. A recovery fidelity of $99.03 \%$ under the effect of temperature inhomogeneity is achieved, the recovered image, in this case, can be seen in Fig. 9(e). Since the overall accuracy of the model is pretty good for the clear and turbid water, and in the presence of temperature inhomogeneity, we turn our attention to the bubbly case where the accuracy is $90.27 \%$. In fact, out of 5775 images in the bubbly test set, the network misclassified 562 images. To emphasize the need for $\mathrm{CNN}$ for the mode detection task, we plot in Fig. 10 examples of modes that are challenging to be identified with the naked eye, and were correctly predicted by the proposed CNN. To have a better idea of the misclassified images, we plot in Fig. 11 a set of these images where we indicate the predicted and true mode of each image. As we can see, most of these images are difficult to classify since some "petals" are missing. Upon inspecting the images, we noticed that there exists up to 310 images (which accounts to $55 \%$ of misclassified modes on the bubbly test set) that are totally obscure (similar to Fig. 12), which may explain why the model accuracy for the bubbly case is around $90 \%$ (compared to $96 \%$ on the test set excluding these images). 


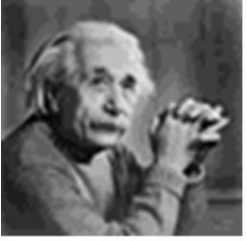

(a)

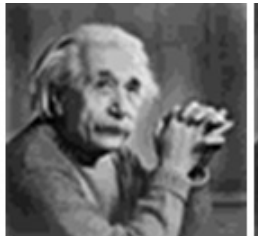

(b)

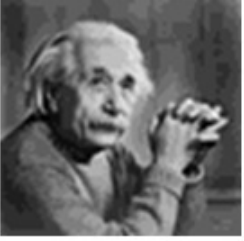

(c)

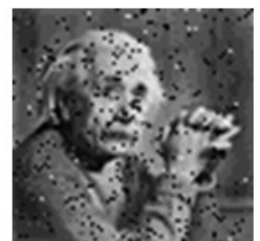

(d)

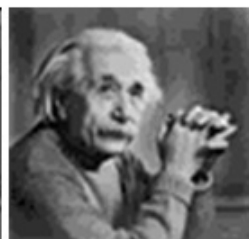

(e)
Fig. 9: (a) Original image. Recovered image after propagation through (b) clear water, (c) turbid water, (d) a bubbly channel, and (e) a channel with temperature in-homogeneity.

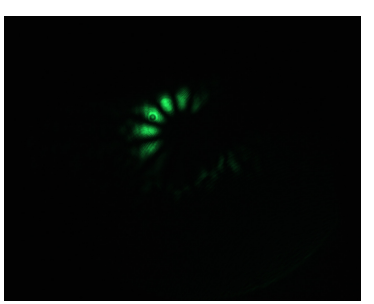

(a) Mode $\mathrm{M}_{6}$

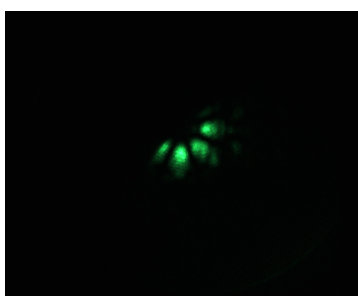

(c) Mode $\mathrm{M}_{4}$

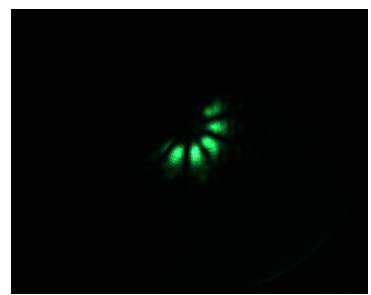

(b) Mode $\mathrm{M}_{5}$

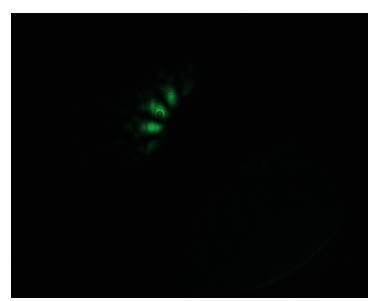

(d) Mode $\mathrm{M}_{5}$
Fig. 10: Examples of correctly identified mode patterns in a bubbly channel.

\section{PRACTICAL IMPLEMENTATION}

One of the main limiting factors of our demonstrations is the switching speed of the used liquid-crystal-based SLM, which is limited to $60 \mathrm{~Hz}$. A faster generation method is possible using commercially available SLMs based on the use of digital micro-mirror devices (DMDs) [55]. Using the multimode encoding technique that we proposed in III-B2 can increase the transmission rate by having multiple spatially separated patterns on the same detector instead of only one. The use of an SLM to dynamically generate the modes may not be practical for out-of lab experiments involving many communicating terminals since it is bulky and a strict alignment between the laser and the SLM must always be maintained. Therefore, the practical implementation of such a communication system for IoUT applications should be

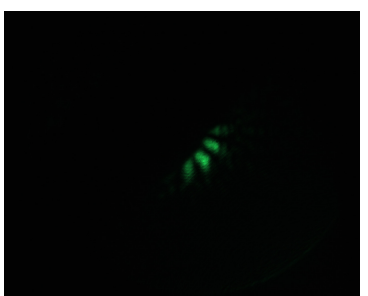
Predicted: Mode $\mathrm{M}_{6}$

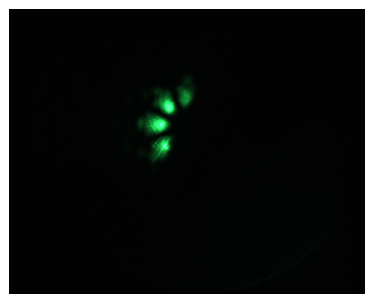

(c) True: Mode $\mathrm{M}_{4}$, Predicted: Mode $\mathrm{M}_{2}$ (a) True: Mode $\mathrm{M}_{7}$,

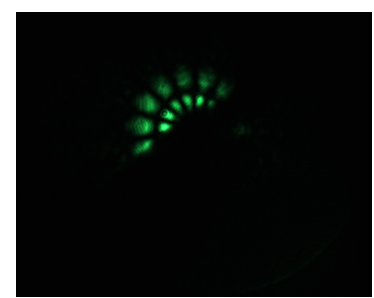

(b) True: Mode $\mathrm{M}_{15}$, Predicted: Mode $\mathrm{M}_{14}$

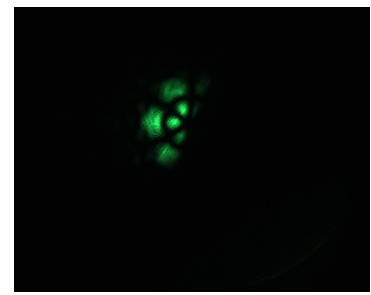

(d) True: Mode $\mathrm{M}_{11}$ Predicted: Mode $\mathrm{M}_{10}$
Fig. 11: Examples misclassified mode patterns in a bubbly channel.

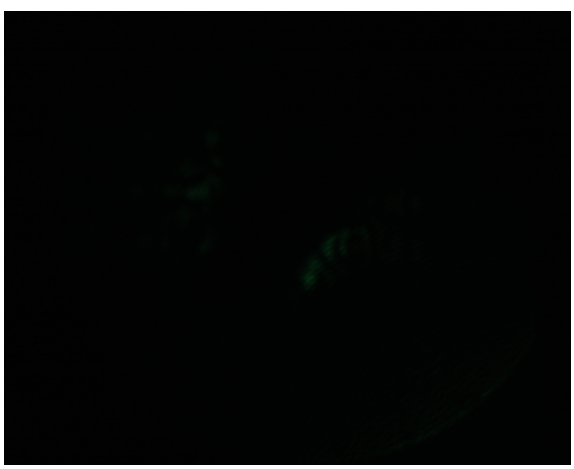

Fig. 12: Obscure pattern recorded by the camera under a bubbly channel.

performed using compact generation devices such as lasers with customized light outputs. 3-D microscale spiral phase plates can also be useful for the design of a mode index modulation-based transmitter [14], [56]. The small foot-print of such nearly-lossless components allows the integration in fast laser switches that could generate high purity modes. A rapid-frame rate camera is also needed to capture the beams in a quick manner and increase the detection bandwidth. High-speed cameras are existing in the market and, if used, can significantly increase the communication rate. The detection sensitivity of the camera can also extend the range of communication.

Instead of only using experimental beam profiles to train the $\mathrm{CNN}$, it is also possible to rely on numerically generated beams that can be obtained when implementing the numerical model that we described in III-C.

We note that the proposed technique can also serve as a tool for light detection and ranging (LIDAR) applications. The idea is to train the CNN with different beam shapes at different distances, which help to localize users in harsh 
underwater environments. Another potential application of our proposed communication scheme is the quantum key distribution $(\mathrm{QKD})$, which has recently attracted considerable attention [57].

\section{CONCLUSION}

In this work, we demonstrated a versatile technique to encode information using the spatial structure of imposed LG modes incorporating the azimuthal and radial indices through an underwater channel. The detection of the LG modes was performed using a convolutional neural network through different kinds of underwater turbulence. The deep learning algorithm was trained using the mode profiles under different channel conditions. Future research directions will involve encoding data over a more significant number of spatial modes from different basis including the Ince-Gaussian and Hermite-Gaussian mode families.

\section{ACKNOWLEDGMENT}

The authors would like to thank Andrea Bertoncini for the experimental setup illustration and the fruitful discussions.

\section{REFERENCES}

[1] M. Chitre, S. Shahabudeen, and M. Stojanovic, "Underwater acoustic communications and networking: Recent advances and future challenges," Mar. Technol. Soc. J., vol. 42, no. 1, pp. 103-116, 2008.

[2] T. Oberg, B. Nilsson, N. Olofsson, M. L. Nordenvaad, and E. Sangfelt, "Underwater communication link with iterative equalization," in Proc. IEEE OCEANS Conf., pp. 1-6, 2006.

[3] A. N. Radford, E. Kerridge, S. D. Simpson, "Acoustic communication in a noisy world: Can fish compete with anthropogenic noise?," Behav. Ecol., vol. 25, no. 5, pp. 1022-1030, Sept.-Oct. 2014.

[4] H. Kaushal and G. Kaddoum, "Underwater optical wireless communication," IEEE Access, vol. 4, pp. 1518-1547, 2016.

[5] H. M. Oubei, J. R. Duran, B. Janjua, H.-Y. Wang, C.-T. Tsai, Y.-C. Chi, T. K. Ng, H.-C. Kuo, J.-H. He, M.-S. Alouini, G.-R. Lin, and B. S. Ooi,, "4.8 Gbit/s 16-QAM-OFDM transmission based on compact 450-nm laser for underwater wireless optical communication," Opt. Express, vol. 23, pp. 23302-23309, 2015.

[6] X. Liu, S. Yi, X. Zhou, Z. Fang, Z.-J. Qiu, L. Hu, C. Cong, L. Zheng, R. Liu, and P. Tian, "34.5 m underwater optical wireless communication with $2.70 \mathrm{Gbps}$ data rate based on a green laser diode with NRZ-OOK modulation," Opt. Express, vol. 25, pp. 27937-27947, 2017.

[7] J. I. d. O. Filho, A. Trichili, B. S. Ooi, M. Alouini, and K. N. Salama, "Toward Self-Powered Internet of Underwater Things Devices," IEEE Commun. Mag., vol. 58, no. 1, pp. 68-73, Jan. 2020.

[8] N. Saeed, A. Celik, T. Y. Al-Naffouri, M.-S. Alouini, "Underwater optical wireless communications, networking, and localization: A survey," Ad Hoc Netw., vol. 94, 101935, 2019.

[9] J. Baghdady, K. Miller, K Morgan, M. Byrd, S. Osler, R. Ragusa, W. Li, B. M. Cochenour, and E. G. Johnson, "Multi-gigabit/s underwater optical communication link using orbital angular momentum multiplexing," Opt. Express, vol. 24, pp. 9794-9805, 2016.

[10] Y. Ren, L. Li, Z. Wang, S. M. Kamali, E. Arbabi, A. Arbabi, Z. Zhao, G. Xie, Y. Cao, N. Ahmed, Y. Yan, C. Liu, A. J. Willner, S. Ashrafi, M. Tur, A. Faraon, and A. E. Willner, "Orbital angular momentumbased space division multiplexing for high-capacity underwater optical communications," Sci. Rep., vol. 6, 33306, 2016.

[11] Y. Zhao, J. Xu, A. Wang, W. Lv, L. Zhu, S. Li, and J. Wang, "Demonstration of data-carrying orbital angular momentum-based underwater wireless optical multicasting link," Opt. Express, vol. 25, pp. 2874328751, 2017.

[12] A. Trichili, K.-H. Park, M. Zghal, B. S. Ooi, M.-S. Alouini, "Communicating using spatial mode multiplexing: Potentials, challenges and perspectives," IEEE Commun. Surv. Tutor, vol. 21, no. 4, pp. 3175-3203, Fourthquarter 2019.
[13] T. Mhlanga, A. Trichili, A. Dudley, D. Naidoo, M. Zghal, A. Forbes, "Digital holograms for laser mode multiplexing," Proc. SPIE 9194, Laser Beam Shaping XV, 91941B, 2014.

[14] G. Ruffato, M. Massari, and F. Romanato, "Generation of high-order Laguerre-Gaussian modes by means of spiral phase plates," Opt. Lett., vol. 39, pp. 5094-5097, 2014.

[15] I. A. Litvin, A. Dudley, F. S. Roux, and A. Forbes, "Azimuthal decomposition with digital holograms," Opt. Express, vol. 20, pp. 10996 11004, 2012.

[16] A. Trichili, C. Rosales-Guzmán, A. Dudley, B. Ndagano, A. Ben Salem, M. Zghal, and A. Forbes, "Optical communication beyond orbital angular momentum," Sci. Rep., vol., 6 Art. No. 27674, 2016.

[17] D. Fu, Y. Zhou, R. Qi, S. Oliver, Y. Wang, S. M. H. Rafsanjani, J. Zhao, M. Mirhosseini, Z. Shi, P. Zhang, and R. W. Boyd, "Realization of a scalable Laguerre-Gaussian mode sorter based on a robust radial mode sorter," Opt. Express, vol. 26, pp. 33057-33065, 2018.

[18] N. K. Fontaine, R. Ryf, H. Chen, D. T. Neilson, K. Kim, and J. Carpenter, "Laguerre-Gaussian mode sorter," Nat. Commun., vol. 10, Art. No. $1865,2019$.

[19] Y. Zhao, C. Cai, J. Zhang, X. Cao, L. Wang, S. Li, and J. Wang, "Feedback-enabled adaptive underwater twisted light transmission link utilizing the reflection at the air-water interface," Opt. Express, vol. 26, pp. 16102-16112, 2018.

[20] Y. Ren, G. Xie, H. Huang, N. Ahmed, Y. Yan, L. Li, C. Bao, M. P. J. Lavery, M. Tur, M. A. Neifeld, R. W. Boyd, J. H. Shapiro, and A. E. Willner, "Adaptive-optics-based simultaneous pre- and postturbulence compensation of multiple orbital-angular-momentum beams in a bidirectional free-space optical link," Optica, vol. 1, pp. 376-382, 2014.

[21] H. Huang, Y. Cao, G. Xie, Y. Ren, Y. Yan, C. Bao, N. Ahmed, M. A. Neifeld, S. J. Dolinar, and A. E. Willner, "Crosstalk mitigation in a freespace orbital angular momentum multiplexed communication link using $4 \times 4$ MIMO equalization," Opt. Lett., vol. 39, pp. 4360-4363, 2014.

[22] J. Lu, V. Behbood, P. Hao, H. Zuo, S. Xue, and G. Zhang, " Transfer learning using computational intelligence: A survey," Knowl.-Based Syst., vol. 80, pp. 14-23, 2015.

[23] W. Dai, Q. Yang, G.-R. Xue, and Y. Yu, "Boosting for transfer learning", in Proceedings of the 24th International Conference on Machine Learning, pp. 193-200, New York, ACM, 2007.

[24] K. He, X. Zhang, S. Ren, and J. Sun, "Deep residual learning for image recognition", in IEEE Conference on Computer Vision and Pattern Recognition (CVPR), pp. 770-778, 2016.

[25] M. Krenn, R. Fickler, M. Fink, J. Handsteiner, M. Malik, T. Scheidl, R. Ursin, and A. Zeilinger, "Communication with spatially modulated light through turbulent air across Vienna," New J. Phys., vol. 16, Art. no. $113028,2014$.

[26] M. Krenn, J. Handsteiner, M. Fink, R. Fickler, R. Ursin, M. Malik, and A. Zeilinger, "Twisted light transmission over 143 km," Proc. Natl. Acad. Sci. USA, vol. 113, pp. 13648-13653, 2016.

[27] E. Knutson, S. Lohani, O. Danaci, S. Huver, and R. T. Glasser, "Deep learning as a tool to distinguish between high orbital angular momentum optical modes," Proc. SPIE 9970, 997013, 2016.

[28] T. Doster and Abbie T. Watnik, "Machine learning approach to OAM beam demultiplexing via convolutional neural networks," Appl. Opt., vol. 56, pp. 3386-3396, 2017.

[29] Q. Zhao, S. Hao, Y. Wang, L. Wang, X. Wan, and C. Xu, "Mode detection of misaligned orbital angular momentum beams based on convolutional neural network," Appl. Opt., vol. 57, pp. 10152-10158, 2018.

[30] Q. Tian, Z. Li, K. Hu, L. Zhu, X. Pan, Q. Zhang, Y. Wang, F. Tian, X. Yin, and X. Xin, "Turbo-coded 16-ary OAM shift keying FSO communication system combining the $\mathrm{CNN}$-based adaptive demodulator," Opt. Express, vol. 26, no. 21, 27849-27864, 2018.

[31] L. Allen, M. W. Beijersbergen, R. J. C. Spreeuw, J. P. Woerdman, "Orbital angular momentum of light and the transformation of LaguerreGaussian laser modes", Phys. Rev. A, vol. 45, no. 11, pp. 8185-8189, 1992.

[32] D. K. Woolf, Bubbles in Encyclopedia of Ocean Sciences, pp. 352-357, (Academic Press, 2001).

[33] H. M. Oubei, R. T. ElAfandy, K. H. Park, T. K. Ng, M.-S. Alouini and B. S. Ooi, "Performance evaluation of underwater wireless optical communications links in the presence of different air bubble populations," IEEE Photon. J., vol. 9, no. 2, pp. 1-9, Apr. 2017.

[34] E. Zedini, H. M. Oubei, A. Kammoun, M. Hamdi, B. S. Ooi, and M Alouini, "Unified statistical channel model for turbulence-induced fading in underwater wireless optical communication systems," IEEE Trans. Commun., vol. 67, no. 4, pp. 2893-2907, Apr. 2019. 
[35] Y. Zhao, A. Wang, L. Zhu, W. Lv, J. Xu, S. Li, and J. Wang, "Performance evaluation of underwater optical communications using spatial modes subjected to bubbles and obstructions," Opt. Lett., vol. 42, pp. 4699-4702, 2017.

[36] M. Cheng, L. Guo, J. Li, Q. Huang, Q. Cheng, and D. Zhang, "Propagation of an optical vortex carried by a partially coherent LaguerreGaussian beam in turbulent ocean," Appl. Opt. vol. 55, pp. 4642-4648, 2016.

[37] W. Wang, P. Wang, T. Cao, H. Tian, Y. Zhang and L. Guo, "Performance investigation of underwater wireless optical communication system using M-ary OAMSK modulation over oceanic turbulence," IEEE Photon. J., vol. 9, no. 5, pp. 1-15, Art no. 7905315, Oct. 2017.

[38] V. V. Nikishov, V. I. Nikishov, "Spectrum of turbulent fluctuations of the sea-water refraction index," Int. J. Fluid Mech. Res., vol. 27, 82-98, 2000.

[39] R. G. Lane, A. Glindemann, and J. C. Dainty, "Simulation of a Kolmogorov phase screen," Waves Random Media, vol. 2, pp. 209-224, 1992.

[40] W. S. McCulloch, and W. Pitts, "A logical calculus of the ideas immanent in nervous activity," B. Math. Biol., vol. 5, no. 4, pp. 115-133, 1943.

[41] Y. Lecun, L. Bottou, Y. Bengio, and P. Haffner, "Gradient-based learning applied to document recognition," Proc. IEEE, vol. 86, no. 11, pp. 22782324, Nov. 1998.

[42] Y. Le Cun, B. Boser, J. S. Denker, R. E. Howard, W. Habbard, L. D. Jackel, and D. Henderson, "Handwritten digit recognition with a back-propagation network," in Advances in neural information processing systems 2, David S. Touretzky (Ed.). Morgan Kaufmann Publishers Inc., San Francisco, CA, USA 396-404, 1990.

[43] D. Ciresan, U. Meier, and J. Schmidhuber, "Multi-column deep neural networks for image classification", in IEEE Conference on Computer Vision and Pattern Recognition, 2012.

[44] D. Ciresan, U. Meier, J. Masci, L. M. Gambardella, and J. Schmidhuber, "Flexible, high performance convolutional neural networks for image classification", in Proceedings of the 22nd International Joint Conference on Artificial Intelligence, vol. 2, pp. 1237-1242, 2011.

[45] S. Lawrence, C. L. Giles, A. C. Tsoi, and A. D. Back, "Face recognition: A convolutional neural network approach," in IEEE Trans. Neural Netw., vol. 8, 1997.

[46] O. Russakovsky, et al., "ImageNet large scale visual recognition challenge," in Int. J. Comput. Vis., 2014

[47] D. Scherer, A. Müller, and S. Behnke, "Evaluation of pooling operations in convolutional architectures for object recognition," in Proceedings of the 20th international conference on Artificial neural networks: Part III (ICANN'10), Berlin, Heidelberg, 92-101, 2010.

[48] P. J. Werbos, "Beyond Regression: New Tools for Prediction and Analysis in the Behavioral Sciences," PhD thesis, Harvard University, 1974.

[49] Y. Le Cun, D. Touresky, G. Hinton, and T. Sejnowski, "A theoretical framework for back-propagation," in Proceedings of the Connectionist Models Summer School, pp. 21--28, CMU, Pittsburgh, Pa : Morgan Kaufmann, 1988

[50] J. Deng, W. Dong, R. Socher, L.-J. Li, K. Li, and L. Fei-Fei, "ImageNet: a large-scale hierarchical image database," in IEEE Conference on Computer Vision and Pattern Recognition (CVPR), pp. 248-255, 2009.

[51] J. Howard et al. "Fastai," 2018. Software available from https://github. $\mathrm{com} /$ fastai/fastai.

[52] A. Paszke et al., "Automatic Differentiation in PyTorch," NIPS Autodiff Workshop, 2017. Software available from https://pytorch.org/.

[53] L. N. Smith, "Cyclical learning rates for training neural networks," in IEEE Winter Conference on Applications of Computer Vision (WACV), Santa Rosa, CA, pp. 464-472, 2017.

[54] L. N. Smith, "A disciplined approach to neural network hyperparameters. Part 1: Learning rate, batch size, momentum, and weight decay," arXiv preprint, arXiv1803.09820, 2018.

[55] S. Turtaev, I. T. Leite, K. J. Mitchell, M. J. Padgett, D. B. Phillips, and T. Čižmár, "Comparison of nematic liquid-crystal and DMD based spatial light modulation in complex photonics," Opt. Express, vol. 25, pp. 29874-29884, 2017.

[56] E. Stegenburgs, A. Bertoncini, A. Trichili, M. S. Alias, T. K. Ng, M.-S. Alouini, C. Liberale, and B. S. Ooi, "Near-infrared OAM communication using 3D-printed micro-scale spiral phase plates," IEEE Commun. Mag., vol. 57, no. 8, pp. 65-69, Aug. 2019.

[57] F. Bouchard, A. Sit, F. Hufnagel, A. Abbas, Y. Zhang, K. Heshami, R. Fickler, C. Marquardt, G. Leuchs, R. w. Boyd, and E. Karimi, "Quantum cryptography with twisted photons through an outdoor underwater channel," Opt. Express, vol. 26, pp. 22563-22573, 2018.

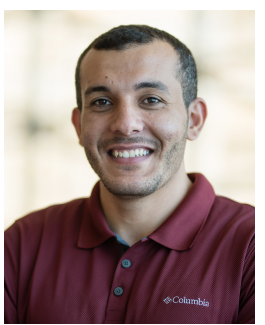

Abderrahmen Trichili received his Diplôme d'Ingénieur and Ph.D. degree in information and communication technology from l'Ecole Supérieur des Communications de Tunis (SUP'COM, Tunisia) in 2013 and 2017, respectively. He is currently a postdoctoral fellow in CEMSE at King Abdullah University of Science and Technology (KAUST). His current areas of interest include space-division multiplexing, orbital angular momentum multiplexing, free-space optical communication, and underwater wireless optical communication systems.

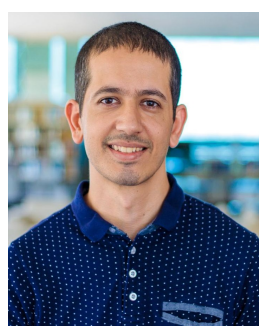

Chaouki Ben Issaid obtained his engineering degree with majors in Economics and Financial Engineering from Ecole Polytechnique de Tunisie (EPT) in 2013. Later on, he obtained his Master in Applied Mathematics and Computational Science (AMCS) from KAUST in 2015 and the Ph.D. degree in Statistics in 2019. His research focus lies in the area of machine learning with applications to wireless communication systems performance.

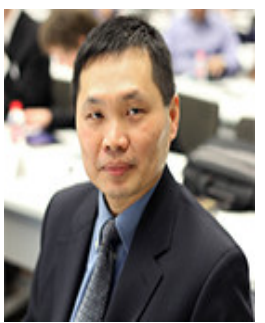

Boon S. Ooi is a Professor of Electrical Engineering at KAUST. He is also the Director of KACST Technology Innovation Center (TIC) for Solid-State Lighting. Professor Ooi received the B.Eng. and Ph.D. degrees in electronics and electrical engineering from the University of Glasgow (Scotland, U.K) in 1992 and 1994, respectively. He joined KAUST from Lehigh University (Pennsylvania, USA), where he held an Associate Professor position and headed the Photonics and Semiconductor Nanostructure Laboratory. In the U.S., his research was primarily funded by the National Science Foundation (NSF) and the Department of Defense and the Army Research Office. In KSA, major funding support for his research is from King Abdulaziz City for Science \& Technology (KACST), Saudi Aramco, SABIC, and Qatar National Research Fund (QNRF).

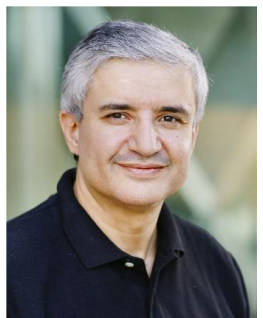

Mohamed-Slim Alouini (S'94-M'98-SM'03-F'09) was born in Tunis, Tunisia. He received the Ph.D. degree in Electrical Engineering from the California Institute of Technology (Caltech), Pasadena, CA, USA, in 1998. He served as a faculty member in the University of Minnesota, Minneapolis, MN, USA, then in the Texas A\&M University at Qatar, Education City, Doha, Qatar before joining King Abdullah University of Science and Technology (KAUST), Thuwal, Makkah Province, Saudi Arabia as a Professor of Electrical Engineering in 2009. His current research interests include modeling, design, and performance analysis of wireless communication systems. 